\title{
Aproveitamento de resíduos da filetagem de tilápia na produção de patê com adição de óleo essencial de orégano
}

Use of tilápia fillet residues in pâté with the addition of oregano essential oil

Utilización de residuos de filete de tilapia em la producción de paté com la adición de aceite esencial de orégano

\section{Resumo}

Foram desenvolvidas 4 formulações de patê utilizando o resíduo da filetagem de tilápia com adição de óleo de orégano: controle sem adição de óleo; 0,03\%; 0,06\% e 0,09\% de óleo. Realizou-se análises de composição centesimal, $\mathrm{pH}, \mathrm{a}_{\mathrm{w}}$, cor e oxidação lipídica (TBARS). Todas as amostras estavam dentro dos padrões físico-químicos estipulados pela legislação brasileira e apresentaram diminuição do $\mathrm{pH}$ durante o período de armazenamento de 90 dias sob refrigeração. Não houve diferença significativa no parâmetro $L^{*}$ e os valores de a* apresentaram uma diminuição durante o armazenamento. Em relação a oxidação lipídica a amostra 0,09\% foi semelhante ao controle. Nesse sentido, conclui-se que a produção de patê com adição de óleo de orégano pode ser uma alternativa para o uso do resíduo da indústria de beneficiamento de tilápia.

Palavras-chave: Óleo essencial; Tilápia; Co-produto; Patê.

\section{Abstract}

Four pâté formulations were developed using the tilapia fillet residue with the addition of oregano oil: control without oil addition; $0.03 \% ; 0.06 \%$ and $0.09 \%$ oil. Analyzes of centesimal composition, $\mathrm{pH}, \mathrm{a}_{\mathrm{w}}$, color and lipid oxidation (TBARS) were performed. All samples were in accordance with Brazilian legislation for physical-chemical standards and showed a decrease in $\mathrm{pH}$ during the storage period under refrigeration. There was no significant difference in the $\mathrm{L}^{*}$ and the values of a* showed a decrease during storage. Regarding lipid oxidation, the sample $0.09 \%$ was similar to 
the control. In this sense, it is concluded that the production of pâté with the addition of oregano oil may be an alternative for the use of the waste from the tilapia processing industry.

Keywords: Essential oil; Tilapia; Co-product; Pâté.

\section{Resumen}

Se desarrollaron cuatro formulaciones de paté utilizando el residuo de filete de tilapia con la adición de aceite de orégano: control sin adición de aceite; $0,03 \% ; 0,06 \%$ y $0,09 \%$ de aceite. Se realizaron análisis de composición centesimal, $\mathrm{pH}, \mathrm{a}_{\mathrm{w}}$, color y oxidación de lípidos (TBARS). Todas las muestras estuvieron dentro de los estándares físico-químicos estipulados por la legislación brasileña y mostraron una disminución del pH durante el período de almacenamiento de 90 días en refrigeración. No hubo diferencia significativa en el parámetro $\mathrm{L} * \mathrm{y}$ los valores de a * mostraron una disminución durante el almacenamiento. En cuanto a la oxidación de lípidos, la muestra al 0.09\% fue similar al control. En este sentido, se concluye que la producción de paté con la adición de aceite de orégano puede ser una alternativa para el aprovechamiento de los desechos de la industria procesadora de tilapia.

Palabras clave: Aceite esencial; Tilapia; Coproducto; Paté.

\section{Introdução}

O interesse da indústria em relação ao pescado vem sendo despertado à medida que o perfil do consumidor passa por mudanças, juntamente com a demanda de produtos alimentícios provenientes da pesca que cresce a cada dia. A tilapicultura no ano de 2015 representava 45,4\% da despesca nacional, contudo, em 2018 os valores chegaram a $60 \%$ do total produzido pela piscicultura (IBGE, 2018). Entretanto, conforme cresce a produção de pescado e a busca por esses alimentos, é notório a geração de resíduos que crescem de maneira proporcional. O peixe quando eviscerado apresenta 8 a $16 \%$ de resíduo, já no processo de filetagem esses valores chegam a 60 a 72\%, e quanto à tilapicultura estima-se que o resíduo gerado é de $64 \%$ (Moraes, 2021).

Desta forma, percebe-se o grau de importância de se desenvolver tecnologias para o aproveitamento desses resíduos, visando melhorar a sua aplicação no mercado e assim, aumentar o aproveitamento integral do pescado, promovendo um maior retorno financeiro e sustentável a piscicultura. O patê desenvolvido com os resíduos da filetagem de tilápia pode ser considerado uma fonte de alimento alternativo, apresentando um grande potencial econômico e aplicação social, visto que estes resíduos são pouco aproveitados, mesmo sendo de grande qualidade nutricional (Matiucci et al., 2020).

Uma maneira de impedir o relevante potencial oxidante dos produtos à base de peixe, seria utilizar antioxidantes naturais. $\mathrm{O}$ objetivo dos antioxidantes é fazer com que a deterioração oxidativa dos alimentos não ocorra, sendo que geralmente os mais utilizados pela indústria são os sintéticos, porém atualmente surgiu um aumento na procura pelos antioxidantes naturais, tal como os óleos essenciais (Fernandes et al., 2018). Conforme o estudo realizado por Del Ré e Jorge (2012), a utilização de orégano (Origanum vulgare L.) é eficaz em todas as fases da oxidação, o que segundo De Morais et al. (2009) está relacionado à presença de flavonóides, catequinas e outros compostos fenólicos.

Sendo assim, o objetivo do presente trabalho foi elaborar patês a partir de resíduos gerados durante o processo de filetagem de tilápia com inclusão de óleo essencial de orégano e avaliar a composição centesimal, $\mathrm{pH}, \mathrm{a}_{\mathrm{w}}$, cor instrumental e oxidação lipídica dos produtos.

\section{Metodologia}

\subsection{Elaboração dos patês}

Os patês foram produzidos no Laboratório de Engenharia de alimentos da Universidade Estadual de Maringá-UEM/PR. As aparas de filetagem de tilápia do Nilo (Oreochromis niloticus) congeladas foram adquiridas na empresa SmartFish situada na cidade de Rolândia, Paraná/Brasil, foram moídas $(3 \mathrm{~mm}$ ) e utilizadas na preparação dos patês. Foram desenvolvidos 4 tratamentos: $\mathrm{C}$ (controle) sem óleo de orégano, com adição de $0,03 \%$ de óleo $(0,03 \%)$, com adição de $0,06 \%$ de óleo $(0,06 \%)$ e com adição de $0,09 \%$ de óleo $(0,09 \%)$. Os ingredientes utilizados nas formulações são de acordo com Matiucci et al. (2019) 
com adaptações e estão apresentados na Tabela 1. Para a produção dos patês os ingredientes foram misturados no cutter até a formação de uma pasta, após, envazados em potes de vidro esterilizados, fechados com tampa e pasteurizados em autoclave por 30 minutos a $78^{\circ} \mathrm{C}$. Após, os produtos foram resfriados e armazenados em geladeira $\left( \pm 7^{\circ} \mathrm{C}\right)$ até a realização das análises.

Tabela 1. Formulações dos patês.

\begin{tabular}{|c|c|c|c|c|}
\hline \multirow{3}{*}{ Ingredientes } & \multicolumn{4}{|l|}{ Tratamentos } \\
\hline & Controle & $0,03 \%$ & $0,06 \%$ & $0,09 \%$ \\
\hline & \multicolumn{4}{|l|}{ Quantidade (\%) } \\
\hline Aparas de tilápia & 58 & 58 & 58 & 58 \\
\hline Gordura hidrogenada & 22,34 & 22,34 & 22,34 & 22,34 \\
\hline Água & 8,86 & 8,86 & 8,86 & 8,86 \\
\hline Gelo & 6,26 & 6,26 & 6,26 & 6,26 \\
\hline Proteína texturizada & 1,34 & 1,34 & 1,34 & 1,34 \\
\hline \multicolumn{5}{|l|}{ de soja } \\
\hline Sal & 0,71 & 0,71 & 0,71 & 0,71 \\
\hline Glutamato monossódico & 0,45 & 0,45 & 0,45 & 0,45 \\
\hline Sais de cura & 0,06 & 0,06 & 0,06 & 0,06 \\
\hline Açúcar & 0,36 & 0,36 & 0,36 & 0,36 \\
\hline Maltodextrina & 0,27 & 0,27 & 0,27 & 0,27 \\
\hline Fumaça líquida & 0,27 & 0,27 & 0,27 & 0,27 \\
\hline Eritorbato de sódio & 0,09 & 0,09 & 0,09 & 0,09 \\
\hline Tripolifosfato de sódio & 0,09 & 0,09 & 0,09 & 0,09 \\
\hline Corante carmim & 0,01 & 0,01 & 0,01 & 0,01 \\
\hline \multicolumn{5}{|l|}{ de cochonilha } \\
\hline Condimentos & 0,89 & 0,89 & 0,89 & 0,89 \\
\hline Total & 100 & 100 & 100 & 100 \\
\hline Óleo essencial* & 0,0 & 0,03 & 0,06 & 0,09 \\
\hline
\end{tabular}

*Óleo essencial de orégano Marca Quinari (Ponta Grossa, Paraná). Fonte: Autores.

\subsection{Composição centesimal, $\mathrm{pH}$ e atividade de água $\left(\mathrm{a}_{\mathrm{w}}\right)$}

A determinação de umidade, cinzas e proteína foi realizada de acordo com a metodologia da AOAC (2005). O teor de lipídios foi determinado pela metodologia de Bligh e Dyer (1959). Os teores de carboidratos foram estimados pelo método de diferença (Brasil, 2003). O pH foi obtido com phmetro digital portátil (Hanna, HI-99163, Romania) equipado com uma sonda de superfície para contato direto com a amostra e calibrado antes e após cada medição usando padrões de pH 4.0, 7.0 e 10.0. A $\mathrm{a}_{\mathrm{w}}$ foi determinada em temperatura ambiente $\left(25^{\circ} \mathrm{C}\right)$, utilizando medidor de atividade de água Aqualab 4TE (Meter Group, USA). A composição centesimal foi realizada no dia 0 e o pH e a a foram avaliados nos dias $0,15,30,45,60,75$ e 90 e todas as análises foram realizadas em triplicata.

\subsection{Cor instrumental}

A determinação de cor instrumental foi realizada utilizando colorímetro (Konica Minolta, CR-400, Japan) segundo Meireles et al. (2020). O equipamento foi calibrado utilizando o branco $\left(L^{*}=85,79, a^{*}=-0,45\right.$ e $\left.b^{*}=3,98\right)$ com iluminante D65 
e os resultados foram expressos pelo sistema CIE L* (luminosidade) a* (variação de vermelho a verde) e b* (variação de amarelo a azul). As análises foram avaliadas nos dias $0,15,30,45,60,75$ e 90 e realizadas em triplicata.

\subsection{Oxidação lipídica (TBARS)}

A análise de oxidação lipídica foi realizada de acordo com a metodologia descrita por Raharjo et al. (1992), modificado por Wang et. al. (2002) seguindo recomendações de Shahidi e Synomiecki (1985) no que se refere à adição de sulfanilamida para as amostras que contém nitrito, com algumas adaptações. Foram adicionados $0,5 \mathrm{~mL}$ de BHT (di-terc-butil metil fenol) 0,5\% em um tubo contendo $5 \mathrm{~g}$ de amostra. Posteriormente, foram adicionados $18 \mathrm{~mL}$ de TCA (ácido tricloroacético) 5\% e realizada a filtração. Em uma alíquota de $2 \mathrm{~mL}$ do filtrado foram adicionados $2 \mathrm{~mL}$ de TBA (ácido 2-tiobarbitúrico) 0,08 $\mathrm{M}$ e a reação foi conduzida em banho-maria a $80{ }^{\circ} \mathrm{C}$ por $40 \mathrm{~min}$. Após a finalização da reação foi realizada a leitura em espectrofotômetro (Agilent UV-8553) a $531 \mathrm{~nm}$. A quantificação foi realizada frente a uma curva padrão (1. 10 $0^{-8}$ a $10.10^{-8}$ $\mathrm{mol} / \mathrm{mL}$ ) de solução de tetraetoxypropane (TEP). Os resultados foram expressos em miligramas de malonaldeído por quilograma de amostra. As análises foram avaliadas nos dias $0,15,30,45,60,75$ e 90 e todas as análises foram realizadas em triplicata.

\subsection{Análise estatística}

O experimento foi repetido três vezes e a análise dos dados foi realizada através da Anova e teste de Tukey utilizando o programa SAS com significância de $5 \%(\mathrm{p}<0,05)$. Todas as análises foram realizadas em triplicata e os resultados expressos como valores médios e desvio padrão.

\section{Resultados e Discussão}

\subsection{Composição centesimal, pH e atividade de água $\left(\mathbf{a}_{w}\right)$}

Na Tabela 2 são apresentados os valores da composição centesimal dos patês de tilápia com adição de óleo de orégano.

Segundo a legislação brasileira (Brasil, 2003) o patê de pescado deve apresentar teor máximo de umidade de 70\%, lipídios 32\%,10\% de carboidratos e teor de proteína bruta no mínimo de $8 \%$. Portanto, observando os resultados é possível verificar que o patê está em concordância com a legislação. Os valores de umidade variaram entre 46,25 e 49,73, o teor de lipídios entre 13,05 e 14,46, o teor de proteína variou entre 28,51 e 32,65, cinzas variou entre 4,39 e 4,79 e os valores de carboidratos variaram de 1,30 a 3,77. A adição do óleo de orégano não influenciou os teores de cinzas, lipídios, proteína e carboidratos $(p>0,05)$ no entanto, o tratamento $0,06 \%$ apresentou diferença significativa $(p<0,05)$ no teor de umidade. Uma possível explicação para esse fato pode ser a diferença da composição da matéria-prima utilizada na elaboração dos produtos. Lovison (2017), da mesma forma não encontraram diferenças significativas nos teores de lipídios e proteína em patê de frango com adição de óleo de orégano. Os resultados de nosso estudo corroboram com os apresentados por Matiucci et al. (2020) em relação aos teores de umidade, por outro lado, os autores encontraram menores valores de proteína, lipídios e cinzas e maiores de carboidratos. Essas diferenças podem ser devido a composição diferente dos patês em relação ao nosso estudo. 
Tabela 2. Composição centesimal dos patês de tilápia com adição de óleo de orégano.

\begin{tabular}{cccccc}
\hline Tratamentos & Umidade & Cinzas & Lipídios & Proteína & Carboidratos \\
\hline Controle & $48,97 \pm 0,19 \mathrm{~A}$ & $4,79 \pm 0,07$ & $13,68 \pm 0,07$ & $31,26 \pm 1,74$ & $1,30 \pm 1,83$ \\
$0,03 \%$ & $49,73 \pm 0,02 \mathrm{~A}$ & $4,73 \pm 0,08$ & $13,05 \pm 0,25$ & $28,84 \pm 0,35$ & $3,65 \pm 0,38$ \\
$0,06 \%$ & $46,25 \pm 0,03 \mathrm{~B}$ & $4,46 \pm 0,16$ & $14,46 \pm 0,86$ & $32,65 \pm 1,52$ & $2,18 \pm 2,51$ \\
$0,09 \%$ & $49,97 \pm 0,87 \mathrm{~A}$ & $4,39 \pm 0,12$ & $13,36 \pm 0,02$ & $28,51 \pm 0,49$ & $3,77 \pm 1,05$ \\
\hline$p$ valor & 0,002 & 0,335 & 0,140 & 0,057 & 0,490 \\
CV & 1,19 & 3,40 & 4,93 & 5,29 & 80,77 \\
\hline
\end{tabular}

Médias na mesma coluna seguidas de letras maiúsculas diferem entre si pelo teste de Tukey $(\mathrm{p}<0,05)$. Dados expressos em média \pm desvio padrão. Fonte: Autores.

Tabela 3. Valores de $\mathrm{pH}$ dos patês.

\begin{tabular}{lllllll}
\hline \multirow{2}{*}{ Tempo } & \multicolumn{2}{l}{ Tratamento } & \multicolumn{2}{l}{} & \\
\cline { 2 - 5 } & Controle & $0,03 \%$ & $0,06 \%$ & $0,09 \%$ & $p$ valor & CV \\
\hline 0 & $6,26 \pm 0,02 \mathrm{aA}$ & $6,30 \pm 0,04 \mathrm{aA}$ & $6,26 \pm 0,05 \mathrm{aA}$ & $6,35 \pm 0,04 \mathrm{aA}$ & 0,625 & 0,52 \\
15 & $6,15 \pm 0,01 \mathrm{aA}$ & $6,06 \pm 0,13 \mathrm{aB}$ & $6,15 \pm 0,04 \mathrm{aA}$ & $6,14 \pm 0,05 \mathrm{aAB}$ & 0,586 & 21,53 \\
30 & $6,12 \pm 0,06 \mathrm{aA}$ & $6,10 \pm 0,01 \mathrm{aAB}$ & $6,14 \pm 0,03 \mathrm{aA}$ & $6,12 \pm 0,08 \mathrm{aAB}$ & 0,976 & 21,14 \\
45 & $6,11 \pm 0,04 \mathrm{aAB}$ & $6,13 \pm 0,02 \mathrm{aAB}$ & $6,06 \pm 0,02 \mathrm{aA}$ & $6,13 \pm 0,01 \mathrm{aAB}$ & 0,839 & 0,39 \\
60 & $5,76 \pm 0,2 \mathrm{bC}$ & $6,09 \pm 0,02 \mathrm{aAB}$ & $6,13 \pm 0,03 \mathrm{aA}$ & $6,06 \pm 0,03 \mathrm{aB}$ & 0,000 & 2,08 \\
75 & $5,69 \pm 0,29 \mathrm{bC}$ & $6,10 \pm 0,02 \mathrm{aAB}$ & $6,07 \pm 0,03 \mathrm{aA}$ & $6,05 \pm 0,06 \mathrm{aB}$ & 0,000 & 9,36 \\
90 & $5,91 \pm 0,07 \mathrm{aBC}$ & $6,05 \pm 0,06 \mathrm{aB}$ & $6,02 \pm 0,05 \mathrm{aA}$ & $6,02 \pm 0,04 \mathrm{aB}$ & 0,308 & 0,75 \\
\hline$p$ valor & 0,000 & 0,411 & 0,086 & 0,001 & & \\
$\mathrm{CV}$ & 6,38 & 23,69 & 0,96 & 1,15 & & \\
\hline
\end{tabular}

Médias na mesma linha seguidas de letras minúsculas diferem entre si pelo teste de Tukey $(\mathrm{p}<0,05)$. Médias na mesma coluna seguidas de letras maiúsculas diferem entre si pelo teste de Tukey $(p<0,05)$. Dados expresso em média \pm desvio padrão. Fonte: Autores.

A partir da Tabela 3, pode-se notar que não houve diferença significativa $(\mathrm{p}>0,05)$ nos valores de $\mathrm{pH}$ entre os tratamentos em relação ao controle, com exceção dos dias 60 e 75 onde a amostra controle apresentou diferença $(\mathrm{p}<0,05) \mathrm{em}$ relação as demais. Em relação ao período de armazenamento, houve diferença entre os tratamentos ( $<$ < 0.05$)$ sendo que no geral ocorreu uma diminuição dos valores de pH. Segundo Sharma et al. (2017), essa diminuição do pH durante o armazenamento dos produtos cárneos é atribuída a produção de ácido láctico pelas bactérias lácticas presentes na matériaprima. Esse comportamento corrobora com o trabalho de Nielsen et al. (2020) em patês de salmão com redução de sódio, onde também houve diminuição do pH. Segundo as Normas Sanitárias do Instituto Adolf Lutz (1967) o pH do patê ou da pasta deve ser levemente ácido, pois esse fato diminui a probabilidade do desenvolvimento de microrganismos deteriorantes. Em nosso estudo os valores de $\mathrm{pH}$ atendem a essa norma, sendo assim o produto pode apresentar um prazo de vida de prateleira estendido.

$\mathrm{Na}$ Figura 1 são apresentados os valores de $\mathrm{a}_{\mathrm{w}}$ das amostras durante o período de armazenamento de 90 dias sob resfriamento $\left( \pm 7^{\circ} \mathrm{C}\right)$. Os valores de $\mathrm{a}_{\mathrm{w}}$ variaram entre 0,978 e 0,990 sendo que houve diferença significativa $(\mathrm{p}<0,05)$ entre as amostras com adição de óleo de orégano e o controle. Valores semelhantes foram demonstrados por Nielsen et al. (2020) em patê de salmão. Por outro lado, Minozzo et al. (2010) em patês de armado (Pterodoras granulosus) demonstraram valores de $a_{w}$ de 0,944 . Apesar das diferenças significativas entre o controle e os demais tratamentos, estas foram pequenas sendo que 
todos tratamentos apresentaram valores acima de 0,970, ou seja, teores elevados de $\mathrm{a}_{\mathrm{w}}$. A atividade de água $\left(\mathrm{a}_{\mathrm{w}}\right)$ é um parâmetro importante na conservação de alimentos, que serve como um indicador para possíveis alterações de origem microbiológicas e físico-químicas que possam ocorrer nos alimentos. Alimentos com alto valor de atividade de água (maior que 0,85 ) podem apresentar maior proliferação de microrganismos e, portanto, faz-se necessário o seu armazenamento sob temperatura de refrigeração ou congelamento (Jay, 2005).

Figura 1. Valores de $\mathrm{a}_{\mathrm{w}}$ dos patês.

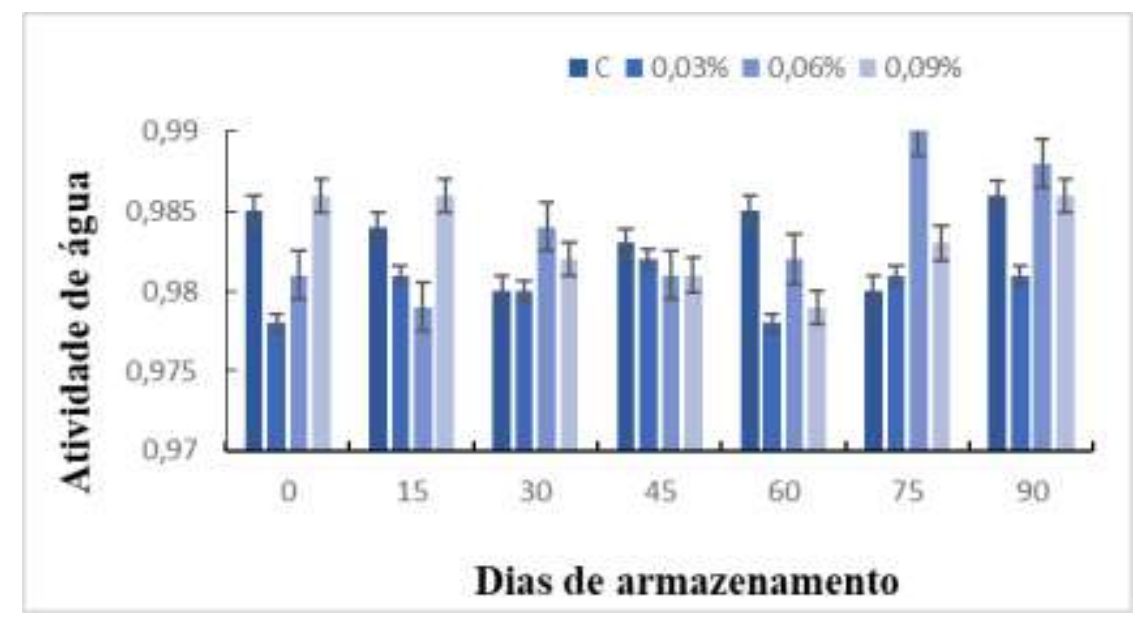

Fonte: Autores.

\subsection{Cor instrumental}

De acordo com os dados apresentados na Tabela 4, não houve diferença significativa $(p>0,05)$ entre as amostras de patê com óleo de orégano e o controle para o parâmetro $L^{*}$. É possível verificar que as médias apresentadas ficaram entre 63,59 e 73,48. Em relação ao período de armazenamento os valores de $L^{*}$ das amostras com adição de óleo foram maiores que o controle no final do período de armazenamento o que corrobora com os resultados de Fernandes et al. (2018) em salsicha de carneiro com adição de extrato de orégano.

Em relação aos valores de $a^{*}$ houve diferença significativa $(p<0,05)$ entre as amostras $0,03 \%$ e $0,06 \%$ em relação ao controle. No geral, ocorreu uma diminuição significativa $(\mathrm{p}<0,05)$ dos valores ao longo do tempo de armazenamento para todos os tratamentos. Essa diminuição nos valores de $\mathrm{a}^{*}$ provavelmente ocorreu devido a reações de oxidação lipídica e oxidação do pigmento nitrosomioglobina, resultando em metamioglobina, o que leva a redução na intensidade da cor (Wójciak et al. 2012). No estudo de Fernandes et al. (2018), utilizando orégano em linguiça, os autores da mesma forma, apresentaram uma diminuição nos valores de $\mathrm{a}^{*}$ durante $\mathrm{o}$ armazenamento. 
Tabela 4. Valores dos parâmetros de cor dos patês.

\begin{tabular}{|c|c|c|c|c|c|c|c|}
\hline \multicolumn{8}{|c|}{ Parâmetros } \\
\hline \multicolumn{2}{|c|}{ de cor } & \multirow{2}{*}{$\begin{array}{c}\text { Controle } \\
69,22 \pm 1,54\end{array}$} & \multirow{2}{*}{$\begin{array}{r}0,03 \% \\
69,30 \pm 2,00 \mathrm{AB}\end{array}$} & \multirow{2}{*}{$\begin{array}{r}0,06 \% \\
69,79 \pm 1,52\end{array}$} & \multirow{2}{*}{$\begin{array}{r}0,09 \% \\
70,90 \pm 1,55\end{array}$} & \multirow{2}{*}{$\begin{array}{l}p \text { valor } \\
0,843\end{array}$} & \multirow{2}{*}{$\begin{array}{c}\text { CV } \\
0,79\end{array}$} \\
\hline $\mathrm{L}^{*}$ & dia 0 & & & & & & \\
\hline & dia 15 & $69,61 \pm 1,91$ & $72,63 \pm 1,22 \mathrm{~A}$ & $72,06 \pm 1,10$ & $69,41 \pm 2,53$ & 0,291 & 2,00 \\
\hline & dia 30 & $68,75 \pm 0,96$ & $69,19 \pm 2,57 \mathrm{AB}$ & $69,56 \pm 1,57$ & $68,61 \pm 2,29$ & 0,968 & 0,50 \\
\hline & $\operatorname{dia} 45$ & $65,79 \pm 3,87$ & $67,95 \pm 1,28 \mathrm{AB}$ & $69,54 \pm 2,55$ & $69,87 \pm 1,85$ & 0,192 & 2,08 \\
\hline & dia 60 & $66,98 \pm 1,96$ & $68,36 \pm 2,45 \mathrm{AB}$ & $68,67 \pm 0,99$ & $69,03 \pm 4,77$ & 0,775 & 0,94 \\
\hline & dia 75 & $63,59 \pm 1,43$ & $65,73 \pm 1,59 \mathrm{AB}$ & $68,90 \pm 2,30$ & $70,50 \pm 1,21$ & 0,005 & 3,75 \\
\hline & dia 90 & $69,18 \pm 1,10$ & $73,48 \pm 6,77 \mathrm{~A}$ & $70,46 \pm 2,01$ & $70,60 \pm 1,81$ & 0,214 & 1,79 \\
\hline \multicolumn{2}{|c|}{$p$ valor } & 0,037 & 0,004 & 0,734 & 0,911 & & \\
\hline \multicolumn{2}{|l|}{$\mathrm{CV}$} & 2,71 & 2,90 & 1,15 & 1,02 & & \\
\hline \multirow[t]{7}{*}{$a^{*}$} & dia 0 & $3,96 \pm 0,45 \mathrm{ab}$ & $0,87 \pm 0,09 c$ & $1,72 \pm 0,14 \mathrm{bc}$ & $4,81 \pm 0,39 a$ & 0,000 & 54,38 \\
\hline & dia 15 & $3,00 \pm 0,58^{\mathrm{a}}$ & $0,19 \pm 0,50 b$ & $0,95 \pm 0,42 \mathrm{ab}$ & $3,44 \pm 040 \mathrm{a}$ & 0,003 & 66,66 \\
\hline & dia 30 & $2,02 \pm 0,57 \mathrm{ab}$ & $-0,64 \pm 0,50 b$ & $0,92 \pm 0,83 \mathrm{ab}$ & $3,04 \pm 0,32 \mathrm{a}$ & 0,003 & 95,79 \\
\hline & $\operatorname{dia} 45$ & $3,10 \pm 0,22 \mathrm{ab}$ & $-0,60 \pm 0,61 c$ & $1,05 \pm 0,27 b c$ & $3,93 \pm 0,60 a$ & 0,000 & 93,55 \\
\hline & dia 60 & $2,63 \pm 0,36 a$ & $-0,63 \pm 0,46 b$ & $0,83 \pm 0,59 a b$ & $2,66 \pm 0,83 a$ & 0,003 & 92,89 \\
\hline & dia 75 & $2,94 \pm 0,62 \mathrm{ab}$ & $0,77 \pm 0,62 b$ & $1,64 \pm 0,26 a b$ & $3,65 \pm 0,63 a$ & 0,027 & 45,86 \\
\hline & dia 90 & $3,43 \pm 1,05 a$ & $-0,10 \pm 0,36 b$ & $0,98 \pm 0,53 \mathrm{ab}$ & $2,65 \pm 0,59 a$ & 0,003 & 71,00 \\
\hline \multicolumn{2}{|c|}{$p$ valor } & 0,653 & 0,570 & 0,957 & 0,356 & & \\
\hline \multicolumn{2}{|l|}{$\mathrm{CV}$} & 13,83 & 22,54 & 26,02 & 16,74 & & \\
\hline \multirow[t]{7}{*}{$b^{*}$} & dia 0 & $15,71 \pm 0,52$ & $13,91 \pm 0,66$ & $14,58 \pm 0,55$ & $15,73 \pm 0,55 \mathrm{~B}$ & 0,050 & 4,93 \\
\hline & dia 15 & $14,79 \pm 0,29$ & $14,83 \pm 0,45$ & $15,02 \pm 0,34$ & $16,50 \pm 0,55 \mathrm{AB}$ & 0,090 & 3,97 \\
\hline & dia 30 & $15,07 \pm 0,31$ & $15,61 \pm 0,75$ & $15,58 \pm 0,38$ & $15,32 \pm 0,32 \mathrm{~B}$ & 0,890 & 1,28 \\
\hline & dia 45 & $15,41 \pm 0,26$ & $15,55 \pm 0,45$ & $14,87 \pm 0,66$ & $16,08 \pm 0,94 \mathrm{~B}$ & 0,490 & 2,19 \\
\hline & dia 60 & $15,18 \pm 041$ & $15,73 \pm 0,65$ & $15,99 \pm 0,45$ & $16,26 \pm 1,38 \mathrm{AB}$ & 0,550 & 2,11 \\
\hline & dia 75 & $16,13 \pm 0,34 b$ & $15,58 \pm 0,38 b$ & $16,36 \pm 1,04 b$ & $18,49 \pm 0,95 \mathrm{aA}$ & 0,000 & 5,56 \\
\hline & dia 90 & $16,67 \pm 0,99 a$ & $13,71 \pm 3,20 b$ & $16,34 \pm 0,63 a$ & $17,03 \pm 0,36 \mathrm{aAB}$ & 0,000 & 6,98 \\
\hline \multicolumn{2}{|c|}{$p$ valor } & 0,208 & 0,057 & 0,113 & 0,002 & & \\
\hline \multicolumn{2}{|l|}{$\mathrm{CV}$} & 3,32 & 4,79 & 3,92 & 4,43 & & \\
\hline
\end{tabular}

Médias na mesma linha seguidas de letras minúsculas diferem entre si pelo teste de Tukey $(\mathrm{p}<0,05)$.

Médias na mesma coluna seguidas de letras maiúsculas diferem entre si pelo teste de Tukey $(\mathrm{p}<0,05)$.

Dados expresso em média \pm desvio padrão. Fonte: Autores.

Na Tabela 4 é possível observar os valores para o parâmetro b* dos patês durante o armazenamento de 90 dias refrigerado que variaram entre 13,91 a 18,49. Houve diferença significativa $(\mathrm{p}<0,05)$ somente após 75 dias de armazenamento, assim como um aumento durante o período de armazenamento para todos os tratamentos. Segundo Rubio 
(2008), um leve aumento nos valores de b* durante a estocagem pode estar ligada a oxidação lipídica, que eleva a intensidade do amarelo através da rancidez.

\subsection{Oxidação lipídica (TBARS)}

A Tabela 5 apresenta os valores de TBARS para os patês de tilápia durante o armazenamento refrigerado. Até o dia 45 não ocorreu diferença significativa ( $p>0,05$ ) entre os tratamentos e o controle, no entanto, a partir do dia 60 as amostras 0,03 e $0,06 \%$ foram diferentes do controle $(\mathrm{p}<0,05)$. Houve aumento nos valores de oxidação durante o período de armazenamento para todos os tratamentos. Esse fato demonstra que a adição de 0,09\% de óleo de orégano na formulação é uma alternativa interessante, pois além do tratamento ser semelhante ao controle o orégano apresenta compostos com ação antimicrobiana, antifúngica e compostos antioxidantes. Esse comportamento também foi observado por Lovison, 2017 em patê de frango com óleo essencial de orégano nanoemulsionado. $\mathrm{O}$ autor mostrou que os teores de TBARS ficaram em média 0,668 mg MDA/kg na primeira semana de armazenamento a $4{ }^{\circ} \mathrm{C}$ e $1,507 \mathrm{mg} \mathrm{MDA} / \mathrm{kg}$ na $16^{\mathrm{a}}$ semana de armazenamento.

Tabela 5. Valores de TBARS dos patês.

\begin{tabular}{lllllll}
\hline \multirow{2}{*}{ Tempo } & \multicolumn{2}{l}{ Tratamentos } & & & \\
\cline { 2 - 6 } & Controle & $0,03 \%$ & $0,06 \%$ & $0,09 \%$ & $p$ pvalor & $\mathrm{CV}$ \\
\hline 0 & $0,18 \pm 0,01 \mathrm{aA}$ & $0,19 \pm 0,01 \mathrm{aC}$ & $0,22 \pm 0,03 \mathrm{aB}$ & $0,21 \pm 0,03 \mathrm{aA}$ & 0,693 & 8,04 \\
15 & $0,21 \pm 0,02 \mathrm{aA}$ & $0,19 \pm 0,01 \mathrm{aC}$ & $0,26 \pm 0,02 \mathrm{aB}$ & $0,21 \pm 0,04 \mathrm{aA}$ & 0,325 & 9,10 \\
30 & $0,18 \pm 0,00 \mathrm{aA}$ & $0,22 \pm 0,02 \mathrm{aBC}$ & $0,24 \pm 0,04 \mathrm{aB}$ & $0,27 \pm 0,06 \mathrm{aA}$ & 0,086 & 11,74 \\
45 & $0,24 \pm 0,02 \mathrm{aA}$ & $0,31 \pm 0,02 \mathrm{aB}$ & $0,23 \pm 0,01 \mathrm{aB}$ & $0,28 \pm 0,07 \mathrm{aA}$ & 0,100 & 11,41 \\
60 & $0,17 \pm 0,03 \mathrm{cA}$ & $0,51 \pm 0,01 \mathrm{aA}$ & $0,22 \pm 0,05 \mathrm{cB}$ & $0,42 \pm 0,06 \mathrm{bB}$ & 0,000 & 41,31 \\
75 & $0,16 \pm 0,02 \mathrm{bA}$ & $0,26 \pm 0,04 \mathrm{aBC}$ & $0,19 \pm 0,02 \mathrm{abB}$ & $0,24 \pm 0,02 \mathrm{abA}$ & 0,038 & 17,49 \\
90 & $0,22 \pm 0,04 \mathrm{cA}$ & $0,32 \pm 0,01 \mathrm{bB}$ & $0,45 \pm 0,01 \mathrm{aA}$ & $0,30 \pm 0,01 \mathrm{bcA}$ & 0,000 & 20,11 \\
\hline$p$ valor & 0,272 & 0,000 & 0,000 & 0,000 & & \\
CV & 13,54 & 28,68 & 21,72 & 17,58 & &
\end{tabular}

Médias na mesma linha seguidas de letras minúsculas diferem entre si pelo teste de Tukey $(\mathrm{p}<0,05)$. Médias na mesma coluna seguidas de letras maiúsculas diferem entre si pelo teste de Tukey $(\mathrm{p}<0,05)$. Dados expresso em média \pm desvio padrão.

Fonte: Autores.

Segundo alguns autores a faixa limite que caracteriza o aparecimento de odor desagradável e de limosidade em produtos cárneos, oriundos da degradação dos lipídios, é de 2,5 mg MDA $/ \mathrm{kg}$, como no presente trabalho os resultados de todos os tratamentos ficaram entre 0,16 a $0,51 \mathrm{mg} \mathrm{MDA} / \mathrm{kg}$ pode-se dizer que os patês estão dentro do valor aceitável para o consumo (Ahmad e Srivastava, 2007; Zhang et al. 2019).

\section{Conclusão}

A utilização dos resíduos de filetagem de tilápia na produção de patês com adição de óleo de orégano foi eficiente pois os produtos apresentaram características positivas em relação ás características fisico-químicas, de cor instrumental e de oxidação lipídica. Essa é uma alternativa ao aproveitamento de um resíduo da indústria de pescados com diminução do impacto ambiental, assim como, uma alternativa para agregar valor aos produtos. 


\section{Agradecimentos}

Os autores agradecem ao Conselho Nacional de Desenvolvimento Científico e Tecnológico (CNPQ) e a Coordenação de Aperfeiçoamento de Pessoal de Nível Superior (CAPES).

\section{Referências}

Ahmad, S., \& Srivastava, P. K. (2007) Quality and shelf life evaluation of fermented sausages of buffalo meat with different levels of heart and fat. Meat Science, 75, 603-603. https://doi.org/ 10.1016/j.meatsci.2006.09.008.

AOAC. (2005). Official Methods of Analysis of AOAC International (17a ed.). USA.

Bligh, E. G. \& Dyer, W. J. (1959). Canadian Journal of Biochemistry and Physiology. Canadian Journal of Biochemistry and Physiology, 37(8).

Brasil. (2003). Ministério da Agricultura. Departamento de Inspeção de Produtos de Origem Animal. Regulamento técnico de identidade e qualidade de patê. http://www.agricultura.govbr/sda/dipoa/anexo1_in_21_2000.htm.

De Morais, S. M., Cavalcanti, E. S., Costa, S. M., \& Aguiar, L. A. (2009). Ação antioxidante de chás e condimentos de grande consumo no Brasil. Brazilian Journal of Pharmacognosy, 19(1B), 315-320. https://doi.org/10.1590/S0102-695X2009000200023.

Del Ré, P. V., \& Jorge, N. (2012). Especiarias como antioxidantes naturais: aplicações em alimentos e implicação na saúde. Revista Brasileira de Plantas Medicinais, 14(2), 389-399. https://doi.org/10.1590/S1516-05722012000200021.

Fernandes, R. P. P., Trindade, M. A., Lorenzo, K. M., \& de Melo, M. P. (2018). Assessment of the stability of sheep sausages with the addition of different concentrations of Origanum vulgare extract during storage. Meat Science, 137, 244-257. https://doi.org/10.1016/j.meatsci.2017.11.018.

Instituto Adolf Lutz - IAL. Normas de qualidade de alimentos. 5. Ofsanpan, 1967.

IBGE (2018). Produção da pecuária municipal 2018. Instituto Brasileiro de Geografia e Estatística-IBGE, 84, 8.

Jay, J. M. Microbiologia de alimentos. (6a ed.), Artemedia. 2005. 711p.

Lovison, M. M. (2017). Óleo essencial de orégano nanoemulsionado: produção, caracterização físico-química, atividade antimicrobiana e antioxidante in vitro e aplicação em patê de frango. Tese de doutorado. Pós-graduação em Engenharia de Alimentos. Universidade de São Paulo. São Paulo-Brasil.

Matiucci, M. A., Souza, M. L. R., Chambo, A. P. S., Vitorino, K. C., Réia, S., \& Verdi, R. (2020). Patês elaborados a partir de resíduos do beneficiamento de tilápia com e sem defumação. Iniciação Científica Cesumar, 21(2), 163-173.https://doi.org/10.17765/1518-1243.2019v21n2p163-173.

Meireles, B. R. L. A, Vitor, R. C. L, Morais, S. K. Q, Barbosa, T. C. M, Costa, S. S \& Fonseca, S. B. (2020). Evaluation of the potential coloring and antioxidant of betalains (Beta vulgaris, L.) in Chicken mortadella. Research, Society and Development, 9(7): 1-22, e237973995. https://rsdjournal.org/index.php/rsd/article/view/3995/3397.

Minozzo, M. G., Waszczynskyj, N., \& Boscolo, W. R. (2010). Production of creamy granulated catfish (Pterodoras granulosus) pate and its microbiological, sensory and physico-chemical characterization. Brazilian Journal of Food Technology, 13(3), 182-188. https:// doi.org/10.4260/BJFT2010130300024.

Moraes, P. S., Engelmann, J. I.; Igansi, A. V.; Cadaval Jr, T. R. S. C., \& Pinto, L. A. A. (2021). Nile tilapia industrialization waste: Evaluation of the yield, quality and cost of the biodiesel production process. Journal of Cleaner Production, 287, 125041. https://doi.org/10.1016/j.jclepro.2020.125041.

Nielsen, T., Mihnea, M., Båth, K., Cunha, S. C., Ferreira, R.. \& Oliveira, H. (2020). New formulation for producing salmon paté with reduced sodium contente. Food and Chemical Toxicology, 143, 111546. https:// doi.org/10.1016/j.fct.2020.111546.

Raharjo, S., Sofos, J. N., \& Schmidt, G. R. (1992). Improved speed, specificity, and limit of determination of an aqueous acid extraction thiobarbituric acid$\mathrm{C}_{18}$ method for measuring lipid peroxidation in beef. Journal of Agricultural and Food Chemistry, 40(11), 2182-2185.

Rubio, B., Martínez, B., García-Cachán, M. D., Rovira, J., \& Jaime, I. (2008). Effect of the packaging method and the storage time on lipid oxidation and colour stability on dry fermented sausage salchichón manufactured with raw material with a high level of mono and polyunsaturated fatty acids. Meat Science, 80, 1182-1187. https:// doi.org/10.1016/j.meatsci.2008.05.012.

Shahidi, F., \& Synomiecki, J. (1985). Protein hidrolyzates from seal meat as phosphate alternatives in food processing applications. Food Chemistry, 60(1), 29-32.

Sharma, H., Mendiratta, S. K., Agrawal, R. K., Gurunathan, K., Kumar, S., \& Singh, T. P. (2017). Use of various essential oils as bio preservatives and their effect on the quality of vacuum packaged fresh chicken sausages under frozen conditions. LWT - Food Science and Technology, 81, 118-127. https://doi.org/10.1016/j.1wt.2017.03.048.

Wang, B., Pace, R. D., Dessai, A. P., Bovell-Benjamin, A., \& Phillips, B. (2002). Modified extraction method for determinating 2-Thiobarbituric acid values in meat with increased specificity and simplicity. Journal of Food Science, 67(8), 2833-2836. https://doi.org/10.1111/j.1365-2621.2002.tb08824.x.

Wójciak, K. M., Dolatowski, Z. J., Kolozyn-Krajewska, D., \& Trzaskowska, M. (2012). The effect of the Lactobacillus casei LOCK0900 probiotic strain on the quality of dry-fermented sausage during chilling storage. Journal of Food Quality, 35, 353-365. https://doi.org/10.1111/j.1745-4557.2012.00458.x.

Zhang, Y., Holman, B. W. B., Ponnampalam, E. N., Kerr, M. G., Bailes, K. L., Kilgannon, A. K., \& Hopkins, D. L. (2019). Understanding beef flavour and overall liking traits using two different methods for determination of thiobarbituric acid reactive substance. Meat Science, 149, 114-119. https://doi.org/10.1016/j.meatsci.2018.11.018. 